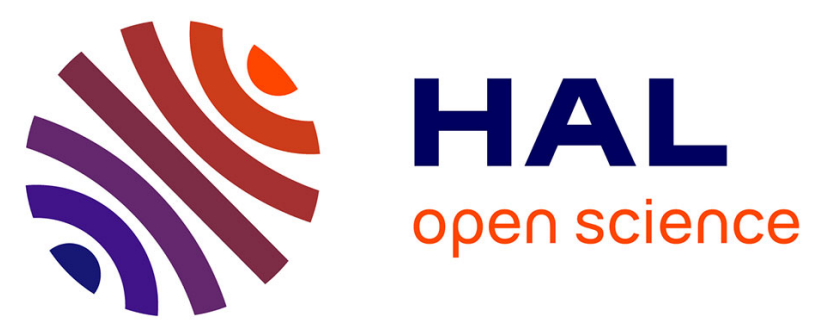

\title{
The mechanism of fatigue crack growth in rubbers under severe loading: the effect of stress-induced crystallization
}

\author{
Jean-Benoit Le Cam, Evelyne Toussaint
}

\section{To cite this version:}

Jean-Benoit Le Cam, Evelyne Toussaint. The mechanism of fatigue crack growth in rubbers under severe loading: the effect of stress-induced crystallization. Macromolecules, 2010, 43 (10), pp.47084714. 10.1021/ma100042n . hal-01131581

\section{HAL Id: hal-01131581 \\ https://hal.science/hal-01131581}

Submitted on 16 Mar 2015

HAL is a multi-disciplinary open access archive for the deposit and dissemination of scientific research documents, whether they are published or not. The documents may come from teaching and research institutions in France or abroad, or from public or private research centers.
L'archive ouverte pluridisciplinaire HAL, est destinée au dépôt et à la diffusion de documents scientifiques de niveau recherche, publiés ou non, émanant des établissements d'enseignement et de recherche français ou étrangers, des laboratoires publics ou privés. 


\title{
The mechanism of fatigue crack growth in rubbers under severe loading: the effect of stress-induced crystallization
}

\author{
J-B. Le Cam ${ }^{l}$, E. Toussaint ${ }^{2}$ \\ 1 Clermont Université, Institut Français de Mécanique Avancée, EA 3867, Laboratoire de Mécanique et \\ Ingénierie, BP 10448, F-63000 CLERMONT-FERRAND \\ 2 Clermont Université, Université Blaise Pascal, EA 3867, Laboratoire de Mécanique et Ingénierie, BP \\ 10448, F-63000 CLERMONT-FERRAND
}

$\underline{\text { lecam@ifma.fr }}$

Received March 16, 2015

The mechanism of fatigue crack growth in rubbers under severe loading

ABSTRACT. This paper deals with the mechanism of fatigue crack growth in natural rubber submitted to severe relaxing loading conditions. In one mechanical cycle under such loading conditions, the high level of stress at the crack tip engenders high crystallinity, which halts crack growth in the plane perpendicular to the loading direction. Consequently, the crack bifurcates. Then the fracture surfaces tear, slide and relax simultaneously along a highly crystallized crack tip to form striations. The higher the stress level, the lower the crack growth in the plane perpendicular to the loading direction and the greater the bifurcation phenomenon. This explains why the striation shape evolves from triangular to lamellar during crack propagation.

KEYWORDS. natural rubber / fatigue striations / stress-induced crystallization / crack bifurcation 


\section{Introduction}

Crack propagation in elastomers is mostly studied through theoretical approaches which do not take into account the physical phenomena involved during the crack propagation process. Thus, these approaches do not satisfactorily predict the effect of microstructure changes due to aging or stressinduced crystallization, and consequently do not adequately predict the crack path. For this purpose, numerous studies investigate the physical phenomena of crack growth. Under quasi-static and repeated loadings (approximately ten cycles for instance), the works of Gent et al. give some important answers concerning the physical mechanisms involved during the crack growth. ${ }^{1-3}$ Under fatigue loadings, crack growth has been studied at the microscopic scale ${ }^{4-8}$ using Scanning Electron Microscopy (SEM). These studies are carried out under relaxing loading conditions, i.e. the loading returns to zero at the end of each mechanical cycle. Moreover, the maximum stress level is moderate in the sense that it does not involve oligocyclic fatigue. Here, the term "Oligocyclic fatigue" is used for severe fatigue loadings leading to a duration life inferior to $10^{4}$ cycles. In such a material and loading conditions, the micromechanism of fatigue crack growth has been identified in carbon black filled natural rubber using an original "microcutting" technique. ${ }^{6}$ This micro-mechanism highlights the fact that the crack tip is composed of elliptical zones separated by ligaments which give rise to a crack tip which is flat but rough. Stress localization at the crack tip favors cavitation and the growth of cavities that weaken the material in the elliptical zones and allow the crack to propagate. Simultaneously, the ligaments do not resist crack propagation, but shrink and join the relaxed zone by generating wrenchings at the fracture surface. Ligaments and elliptical zones are then regenerated. Even if the mechanism proposed by the authors is suitable for moderate loading, it is not sufficient to explain the morphology of the fracture surface when the stress level increases significantly at the crack tip, i.e. for olygocyclic fatigue or for the end of crack propagation under moderate cyclic loading. In these cases, no wrenching is observed at the fracture surface; only striations are observed. The last remark suggests that the mechanism of fatigue 
crack growth changes when the stress increases significantly at the crack tip.

The aim of this paper is to establish the mechanism of fatigue crack growth under severe cyclic loading conditions and to evaluate the contribution of stress-induced crystallization to this mechanism. Even if the phenomenon of stress-induced crystallization has been widely studied under quasi-static loading, ${ }^{9-12}$ its influence on the mechanism of fatigue crack growth under severe fatigue loading has never been investigated at the microscopic scale. For this purpose, uni-axial fatigue tests are performed until failure. Some tests are halted during crack propagation. SEM is employed to examine the fracture surfaces and the stretched crack tip. An original experiment is then carried out in order to investigate in real-time the relative displacement of each crack tip zone when the stress significantly increases. This experiment is expected to provide significant information concerning the role of stress-induced crystallization on the crack growth scenario. Finally, observations and measurements performed are considered to establish the mechanism of fatigue crack growth. A conclusion and some perspectives close the paper.

\section{Experimental Section}

2.1 Materials and samples. The material considered here is a $34 \mathrm{phr}$ carbon black-filled natural rubber. Table I summarizes its chemical composition and some mechanical characteristics. It should be noted that the material formulation is the same as that of the study in reference 3 . Consequently, the same physical mechanisms are induced during crack propagation under moderate loading. The compound was cured for $7 \mathrm{~min}$ and the mold temperature was set to $160^{\circ} \mathrm{C}$. It is referred to as F-NR in the following. The degree of cross-linking, characterized by the number $v$ of moles of cross-links per g, which was estimated using the Mooney elastic coefficient $C_{1}{ }_{1}^{13}$ was found to be equal to $11.3 \mathrm{~mol} / \mathrm{g} \times 10^{-5}$. To overcome aging problems, samples were frozen at $-18^{\circ} \mathrm{C}$ for 48 hours after molding. They were then thawed out for 24 hours before testing. Two sample geometries were used. The first was a classic sample geometry for fatigue tests ${ }^{11}$ and is presented in Figure 1. It is axisymmetrical and is usually 
called a "diabolo" sample. It is adhered on both sides to metallic inserts in order to be clamped in the grips of the testing machine. The second geometry corresponds to a plate of $20 \mathrm{~mm}$ height, $5 \mathrm{~mm}$ width and $2 \mathrm{~mm}$ thick. It is referred to as the "flat" sample in the following and was used to observe the realtime change in the mechanism of fatigue crack growth when the stress level increases at the crack tip.

2.2 Fatigue loading conditions. The uniaxial fatigue tests were first performed with the diabolo samples under uniaxial cyclic prescribed force with a MTS 858 Elastomer Test System testing machine. Table 2 summarizes the fatigue tests performed under relaxing loading conditions, i.e. the minimum value of the cyclic force is equal to zero. Four levels of force were applied using a sinusoidal signal: 300, 500, 600 and 750 N. For each test, three samples were tested and the number of cycles at crack initiation (the occurrence of a self-initiated crack of $2 \mathrm{~mm}$ at the sample surface) and at failure were stored. Three tests were also halted during crack propagation under $500 \mathrm{~N}$ maximum force in order to investigate the crack tip morphology when a change in the mechanism of fatigue crack growth was observed. The frequency was set to limit the rise in surface temperature to $20^{\circ} \mathrm{C}$ and to avoid creating thermal damage in addition to the mechanical damage. These tests were carried out at $23^{\circ} \mathrm{C}$ regulated temperature.

The flat samples were pre-cut with a razor blade and were then tested under the same conditions as the diabolo sample but with a lower prescribed force (between 0 and $15 \mathrm{~N}$ ) to account for the difference in cross-section area between the two sample geometries. When the crack tip becomes similar to that of a typical fatigue crack tip obtained under moderate loading, the sample is clamped in the grips of a microtensile machine (described in the following). It is then stretched to an elongation higher than the maximum measured during the fatigue test in order to observe in real-time the effect of the increase in stress on the crack propagation mechanism.

2.3 Scanning electron microscopy. Photomicrographs were performed with a HITACHI S-3200N model SEM using secondary electrons. Before observation, the elastomer surfaces were cleaned; the specimens were submitted to ultrasounds in a neutral solution. Moreover, the considered material not 
being sufficiently conductive, charge build-up occurs when it is irradiated with the electron beam. Thus, samples were stretched with a rudimentary static tensile apparatus ${ }^{6}$ to a stretch ratio equal to that measured at the last mechanical cycle and the crack tip was coated with a $90 \mathrm{~nm}$-thick layer of gold by vapour deposition to ensure electrical conduction.

2.4 Measurement of the relative displacement field at the crack tip. The measurement was carried out at the crack tip of the notched flat sample while it was stretched using a DEBEN micro-tensile machine. This machine allows the sample to be stretched symmetrically. Figure 2 presents the experimental setup. It consisted of a cooled 12-bit dynamic Sensicam camera with a Questar long distance microscope which enables the observation of zones of $2.7 \times 2 \mathrm{~mm}^{2}$ area. Uniform lighting at the sample surface was ensured by lamps. The charge-coupled device (CCD) of the camera has $1.410^{6}$ joined pixels $(1376 \mathrm{x}$ 1040). The camera was fixed on a multidirectional adjustable support. The relative displacement field at the crack tip was obtained using the Digital Image Correlation (DIC) technique ${ }^{15}$. This consists in correlating the grey levels between two different images of a given zone. Each image corresponds to different stretch ratio levels. This optical technique offers a resolution of 0.03 pixel corresponding to $0.06 \mu \mathrm{m}$ and a spatial resolution (defined as the smallest distance between two independent points) of 16 pixels corresponding to $31 \mu \mathrm{m}$. The software ${ }^{16}$ used for the correlation process was Correli ${ }^{\mathrm{LMT}}$. It should be noted that the stretch ratio level can not be measured using the images of the front of the crack because the image of reference corresponds to the crack closed. For this reason, the experiment was also performed with a second sample for which the CCD camera records images of the side view of the crack during stretching.

\section{Results}

In this section, typical fracture surfaces are first presented by highlighting the change in morphology when the stress significantly increases. Second, halted tests are used in order to observe the crack tip at the first stage of striation formation and to attempt to link the morphology of the crack tip with that of 
the fracture surface. Finally, real-time observations of the highly stretched crack tip are carried out to highlight the contribution of stress-induced crystallization to the mechanism of fatigue crack growth and consequently to explain the formation of striations.

3.1 Typical fracture surface. At the macroscopic scale, cracks always propagate in a plane perpendicular to the applied force direction in a zone close to the middle plane of the sample. Figure 3 summarizes the fracture surface morphology observed. Figure 3(a) is the top view of a typical fracture surface. Five zones are considered to describe it:

- zone A is the initiation zone. It is always located close to the sample surface. This zone is identified by the orientation of wrenchings on the fracture surface, which describe ellipses around this zone. Further investigations using SEM coupled with an Energy Dispersive X-ray Spectrometer (EDXS) have shown that crack initiation is due either to the failure of carbon black agglomerates or to the cavitation in the rubber matrix in the vicinity of the agglomerate poles;

- zone B contains zone A and corresponds to the area where the fatigue crack begins to grow from the critical defect. It forms an elliptical crack tip (due to the surface vicinity) which propagates to the outer surface and simultaneously through the bulk of the sample. The crack grows and generates wrenchings formed by the shrinking of ligaments located at the crack tip;

- zone $\mathrm{C}$ is the zone corresponding to crack propagation through the bulk of the sample. It is covered by wrenchings which increase in size with the increase in stress at the crack tip;

- zone D is composed of striations. No wrenching is observed in this zone. It should be noted that the size of this zone increases with the maximum stress at the crack tip. Thus, zone B is not observed on the fracture surface and striations begin to form around the crack initiation zone. These observations show that fatigue striations are the signature of the increase in stress at the crack tip. Moreover, their shape evolves as the stress increases. As shown in Figure 3(b), two types of striation shape are observed: triangular and lamellar striation shapes. They occur successively with the increase in stress. Each of them is smooth. As shown in Figure 3(c), the triangular shape is composed of two perpendicular 
surfaces, denoted L and $\mathrm{P}$ in the following. As the stress level increases, the area of surface L increases and that of the perpendicular surface $\mathrm{P}$ decreases until it becomes a line, consequently forming lamellar striations. Moreover, cracks are observed between the striations. This phenomenon resembles crack bifurcation or micro-branching. The fact that this crack is the continuation of surface $\mathrm{P}$ indicates that this surface corresponds to crack propagation. At this stage of the present observations, a question of importance arises: do these surfaces form in one or several cycles? This question is discussed in the following. Figure 3(d) presents striations that do not form in the same plane. The frontier between them is highlighted with dotted lines in this figure. This indicates that the crack can propagate independently in several zones by forming striations along the tip. When the stress increases at the crack tip, these zones can coalesce, i.e. crack propagates simultaneously through them. Finally, cavities are observed on surface P (Figure 3(b) and Figure 3(d)), but contrary to the case of moderate loading, they do not seem to contribute to the mechanism of crack growth: the crack propagates through them;

- zone E corresponds to the final fracture surface. This zone is smooth and is comparable to the fracture surface obtained for static crack propagation. That is explained by the fact that the stress level is as great as that necessary for static fracture.

As a summary, the observation of fracture surfaces provides relevant information about crack growth, especially the fact that fatigue striations take place under severe loading conditions, but it is not sufficient either to establish the mechanism of fatigue striation formation or to explain the evolution from a triangular to a lamellar striation shape. This last remark motivates the fatigue tests which are halted during crack propagation, especially when fatigue striations begin to form. Thus, using an apparatus to stretch the sample, the crack tip can be observed in-situ. The aim of this experiment is to link the morphology of the crack tip to that of the fracture surface, and more particularly that of the fatigue striations. Thus, it could be possible to explain the change in the fatigue crack growth mechanism between moderate and severe loading.

3.2 Crack tip observation. The observations were carried out with samples from halted fatigue tests for 
which striations begin to form. In order to observe the crack tip morphology, the sample is stretched using a basic apparatus and a gold layer is vapor-deposited at its surface. Figure 4 shows successive magnifications of the crack font. Because of its size, the sample stretched using a basic apparatus undergoes rotation in the SEM chamber. This is the reason why the sample is not exactly aligned with the photomicrograph borders. The four photomicrographs of Figure 4 correspond to successive magnifications of the zone where fatigue striations initiate. Figure 4(a) shows the frontier (dotted line) between the crack tip and the relaxed failed surfaces that form the crack lip. This frontier corresponds to that between Zones C and D in Figure 3(a). Figure 4(b) is the magnification of the outlined zone in Figure 4(a) and shows that the crack tip is composed of large ligaments and elliptical zones. Moreover, fatigue striations are observed at the relaxed failed surface. This seems to indicate that ligaments and elliptical zones take part in the beginning of fatigue striation formation and that the change in the fatigue crack growth mechanism is a continuum phenomenon. The two last photomicrographs highlight the feet of the ligaments and of the elliptical zones whose morphology clearly differs from that observed during the formation of wrenchings ${ }^{7}$ : no micro-cracks are observed but fatigue striations begin to form. This shows that the mechanism of fatigue crack growth under moderate loading is not suitable to describe the formation of fatigue striations under high loading: under moderate loading, cavities grow and weaken the crack tip but under severe loading, the analysis of Figure 3(b) and Figure 3(d) shows that cavities do not grow significantly to allow the crack to propagate. Indeed, the crack propagates through cavities whose size (about $10 \mu \mathrm{m})$ does not exceed significantly that of zinc oxide agglomerates $(5 \mu \mathrm{m}$ maximum). Thus, due to the high stress level, the crack seems to propagate rapidly through the elliptical zones and consequently cavities have no time to grow. Figure 4(d) shows the magnification of the striation formation zone. Striations are identified by the smooth surfaces perpendicular to the plane of crack propagation. In fact, they correspond to the L surfaces. Here, questions of importance arise: Why are smooth surfaces observed perpendicular to the plane of crack propagation? What is the effect of stress-induced crystallization on the mechanism of crack growth? Is the change of mechanism between 
moderate and severe loading continuous? The next experiment aims to answer these questions.

3.3 Real-time observation of crack growth under high stress levels. As mentioned previously, striations are generated under high stress levels at the crack tip. The aim of the present experiment is therefore to investigate the effect of the increase in stress on the mechanism of fatigue crack growth. The experiment consists of cutting the flat sample with a razor-blade and then applying moderate relaxing cyclic loading to propagate the crack. In order to generate a similar stress level at the crack tip as that applied to the diabolo sample, the force varies between 0 and $15 \mathrm{~N}$. As a consequence, only wrenchings are generated on the fracture surface. It should be noted that the number of cycles before stopping the test is set in such a way that the effect of the razor-blade cutting is eliminated. In fact, compared to a self-initiated crack, the crack tip obtained with a razor-blade generates a higher stress concentration. Consequently, the crack rapidly propagates and generates smooth fracture surfaces until a crack tip shape and morphology is obtained which is similar to that obtained without the pre-cut with a razor blade, ${ }^{17,18}$ i.e. with a self-initiated crack.

Figure 5(a) presents the crack tip obtained for the maximum force applied during the fatigue test. As expected by applying such moderate loading conditions, it is composed of ligaments and elliptical zones. It is flat but rough. It should be noted that the stretch ratio level measured at the crack tip equals 2.25. This was established by observing the side view of the crack during stretching and by using the DIC technique to determine the stretch ratio. As previously explained, in such a material, the stretch ratio at which crystallization begins equals 1.64 at ambient temperature. This means that under moderate loading, stress-induced crystallization occurs at the crack tip but does not significantly affect the crack path. Hence, progressively increasing the force applied, and consequently the stress concentration at the crack tip, by increasing the enforced displacement at a strain rate corresponding to $2 \mathrm{~mm} / \mathrm{min}$ leads to an increase in the crystallinity. During stretching, images of the crack tip are stored in order to measure the relative kinematic field and to calculate the deformation level at the crack tip using the Digital Image Correlation (DIC) technique. 
Figure 5(b) shows the evolution of the crack tip morphology during stretching. Ligaments and the elliptical zones are highly stretched and they join the relaxed zone. None of them are regenerated and the crack tip, previously rough, becomes smooth. Figure 5(c) shows the same experiment performed with the diabolo sample. The crack tip obtained is similar. This indicates that the phenomena observed with the flat sample do not depend on the geometry of the sample and are intrinsic to the microstructure of the natural rubber. It should be noted that this image is obtained by SEM analysis. As SEM induces image distortion, optical microscopy is preferred here to estimate the kinematic field by image correlation. Figure 5(d) shows the crack tip morphology obtained at the end of the stretching. To explain the change in the morphology of the crack tip between moderate and severe loadings, it is necessary to establish the relative deformation of the zones that compose the crack tip. For this purpose, the DIC technique is used. Two images are considered: the reference image in Figure 5(b) and the more stretched image in Figure 5(d), obtained by increasing the displacement of the grips. By observing the evolution of the smooth, flat surfaces between the two images, it clearly appears that their area is higher when the sample is stretched and that no new ligament is formed. The question is to know if this is due to the crack that propagates through it or to another phenomenon. For this purpose, the DIC technique is used to measure the relative displacement (and therefore the relative deformation) of the different zones that form at the crack tip.

Figure 6(a) presents the result of the image correlation. The correlation area corresponds to the boxed area in Figure 5(b). The results, in terms of the components of the Green-Lagrange tensor ${ }^{19}$ in the stretching axis (left), are given relative to this boxed area. First of all, three zones can be distinguished in terms of deformation. They correspond to the smooth surfaces, to the ligaments and to the surface between the relaxed zone and the ligaments. To compare the relative deformation (deformation obtained by considering that Figure 5(b) is the reference image) of these zones during stretching, three points are considered, one in each zone; point $\mathrm{A}$ for the ligament, point $\mathrm{B}$ for the smooth surface and point $\mathrm{C}$ for smooth surface between the ligament and relaxed zone. Figure 6(b) gives the relative deformation at 
these points. By considering point $\mathrm{B}$, the deformation of the flat, smooth zone remains close to zero between the two deformation states. In fact, the maximum deformation level is obtained at point $\mathrm{A}$ in the zone that contains the ligament. This zone joins the relaxed zone by tearing and sliding along the smooth, flat surface. This is the reason why compression is detected at point $\mathrm{C}$ between the ligament and the relaxed zones. The fact that the crack no longer propagates from the smooth surfaces and that no deformation is measured for the smooth, flat zone, indicates that the microstructure has changed, i.e. crystallinity has increased and has reinforced the crack tip. This is the reason why the crack does not propagate through it. In the present natural rubber, crystallization begins at a 1.64 stretch ratio, ${ }^{20,21}$ i.e. at a stretch ratio lower than that at the crack tip. This zone is therefore similar to a wall that stops crack propagation and induces crack bifurcation. Moreover, ligaments and elliptical zones are not regenerated. This explains why wrenchings, which are due to the successive shrinking of ligaments, are not observed in the striation zone at the fracture surface (see zone D in Figure 3(a)). This also explains why the surface perpendicular to the direction of crack propagation is smooth: it corresponds to the smooth and flat surface observed.

3.4 Mechanism of fatigue crack growth under severe loading. In this section, the previous observations are considered to establish the scenario of fatigue crack growth under severe relaxing loading conditions. It is described in Figure 7 and Figure 8 through two chronological sketches corresponding to the font and side views of the crack tip, respectively. These two views are necessary to describe precisely the three-dimensional nature of the phenomenon. It should be noted that the chronology of the two figures is different: the step shown in Figure 7(a) does not correspond to that of Figure 8(a). Contrary to Figure 7(a), Figure 8(a) shows the crack in the non-deformed state. In most cases, the formation of striations follows the formation of wrenchings. As the transition between the two fatigue crack growth mechanisms is a continuum phenomenon (see Figure 4(b)), it seems relevant to begin the description of the crack growth mechanism under severe loading by the crack tip composed of elliptical zones separated by ligaments, i.e. obtained under moderate loading. It is presented in Figure 7(a). In this 
case, cavities located behind the crack tip (dotted lines) weaken the material in the elliptical zones by forming thin membranes between them and the crack tip which allow the crack to propagate. Ligaments, which do not resist crack propagation, fail and shrink to form wrenchings at the fracture surface. When the stress increases at the crack tip, the crack propagates rapidly in the centre of the elliptical zones (Figure 7(b)) and cavities do not have enough time to grow and to weaken the material. The surface generated by the crack growth is perpendicular to the loading direction and corresponds to surfaces $\mathrm{P}$ (the grey surfaces in Figure 7(c)). When the stretch ratio is higher at the end of stretching, the crack encounters zones which are much more crystallized than at the beginning of the stretching, and the direction perpendicular to the loading direction is no longer that of lesser energy. Consequently, the crack bifurcates (see Figure 3(c)). Once this new mechanism is established, one cycle is sufficient to form one striation (see Figure 7(c) and Figure 7(d)). Figure 8(b), Figure 8(c) and Figure 8(d) illustrate the fact that with a decrease in the sample cross-section, which leads to an increase in stress at the crack tip and in crystallinity in this zone, the part of the cycle dedicated to propagation in the direction perpendicular to that of loading becomes less and less significant. In other words, the area of surfaces $\mathrm{P}$ is lower. Moreover, as highlighted by the second experiment described in section 3.1, the formation of surface $\mathrm{L}$ is due to both crack bifurcation and the tearing and sliding of the striations along the smooth, flat surface which is highly crystallized. Then, successively generated P surfaces join the relaxed zones and reorient themselves in the loading direction. This mechanism explains the change in the striation shape, the transition between triangular and lamellar striations. Finally, Figure 7(c) and Figure 7(d) illustrate the fact that elliptical zones are not regenerated. This explains why the flat, rough crack tip under moderate loading becomes flat and smooth under severe loading.

It should be noted that in the case of oligocyclic fatigue, striations form around the crack initiation zone and no wrenching is observed. This indicates that only one mechanism is activated: the one of crack growth under severe loading previously described in Figure 7(c) and Figure (d)). 


\section{Conclusion and perspectives}

The mechanism of fatigue crack growth under severe relaxing loading conditions was established using two complementary experiments. The first one consists of observing the stretched crack tip morphology using SEM. The second one enables the relative deformation of each zone at the crack tip to be established using the DIC technique. Results show that under severe loading, the mechanism strongly differs from that of fatigue crack growth under moderate loading. Under severe fatigue loading, the crack propagates by generating only striations; no ligament is regenerated. This phenomenon is due to the fact that the high cristallinity at the crack tip reinforces the material in such a way that the crack can not propagate through it and bifurcates at the microscopic scale. Then the striations formed tear, slide and relax along the smooth surface of the crack tip. This smooth surface joins the relaxed zone and reorients in the direction perpendicular to that of the loading. Thus, striations and consequently microbifurcation are the characteristic phenomena of fatigue cracks. The results obtained in the present study at the microscopic scale present some interesting ways to analyze crack bifurcation occurring under

non-relaxing conditions (the minimum stress level remains positive) at the macroscopic scale ${ }^{14}$. The fact that the force does not remain equal to zero at the end of each cycle prevents crystallites from melting at the crack tip and therefore prevents cracks from propagating. Finally, it is well-known that cristallinity depends on both the stretch ratio and the temperature. This last remark shows the relevancy of investigating the influence of temperature on the mechanism of fatigue crack growth. Further work in this field is currently being envisaged by the authors of this paper.

\section{Acknowledgment}

Authors thank D. Ly for his collaboration.

References and Notes 
(1) Gent, A. N.; Pulford, C. T. R. Journal of Materials Sciences 1984, 19, 3612-3619.

(2) Hamed, G. R.; Kim, H. J.; Gent, A. N. Rubber Chemistry and Technology 1996, 69, 807-818.

(3) Gent, A. N.; Razzaghi-Kashani, M.; Hamed, G. R. Rubber Chemistry and Technology 2003, 76, 122-131.

(4) Fukahori, Y. in Fractography of Rubber Materials; Bhowmick A. K., De S. K., Eds.; Elsevier Applied Science: London and New York, 1991; p71.

(5) Bhowmick, A. K. Rubber Chemistry and Technology 1995, 68, 132-135.

(6) Le Cam, J.-B.; Huneau, B.; Verron, E.; Gornet, L. Macromolecules 2004, 37, 50115017.

(7) Hainsworth, SV. Polymer Testing 2007, 26, 60-70.

(8) Wang, B.; Lu, H.; Kim, G. Mechanics of Materials 2002, 34, 475-483.

(9) Trabelsi, S.; Albouy, P.-A.; Rault, J. Macromolecules 2002, 35, 10054-10061.

(10) Trabelsi, S.; Albouy, P.-A.; Rault, J. Macromolecules 2003, 36, 9093-9099.

(11) Rault, J.; Marchal, J.; Judeinstein, P.; Albouy, P.-A. Macromolecules 2006, 39, 83568368.

(12) Toki, S.; Sics, I.; Ran, S.; Liu, L.; Hsiao, B.; Murakami, S.; Senoo, K.; Kohjiya, S. Macromolecules 2002, 35, 6578-6584.

(13) Treloar, L. R. G. The Physics of Rubber Elasticity, 3rd ed.; Clarendon Press: Oxford, 1975.

(14) Le Cam, J.-B.; Verron E.; Huneau, B. Fatigue and Fracture of Engineering Material \& Structures 2008, 31, 1031-1038.

(15) Sutton, M. A.; Wolters, W. J.; Peters, W. H.; Ranson, W. F.; McNeill, S. R. Image and Vision Computing 1983, 1, 133-139.

(16) Hild, F. Correli ${ }^{\mathrm{LMT}}$ : a software for displacement field measurements by digital image correlation, internal report $n^{\circ} 254,2002$.

(17) Thomas, A. G. Journal of Polymer. Science 31, 1958, 467-480.

(18) Lake, G. J.; Lindley, P. B. Journal ol Applied Polymer Science 9, 1965, 2031-2045. 
(19) Holzapfel, G.A. Nonlinear Solid Mechanics: A Continuum Approach for Engineering. Wiley, New York, 2000.

(20) Le Cam, J.-B.; Toussaint, E. Macromolecules 2008, 41, 7579-7583.

(21) Le Cam, J.-B.; Toussaint, E. Mechanics of Materials 2009, 41, 898-901. 
List of tables

Table 1. Material formulation and mechanical properties

Table 2. Description of the fatigue test 


\section{List of figures}

Figure 1. Diabolo sample.

Figure 2. Real-time measurement of the relative displacement field at the crack tip: experimental set-up.

Figure 3. Description of the fracture surface obtained under relaxing loading conditions: (a) schematic view; (b) triangular and lamellar shapes; (c) crack bifurcation between triangular striations; (d) cavities located on surface L.

Figure 4. Description of the crack tip: (a) frontier between the crack tip and the fracture surface; (b) magnification of the outlined zone of Figure 4(a); (c) magnification of the outlined zone of Figure 4(b); (d) magnification of the outlined zone of Figure 4(c).

Figure 5. Evolution in crack tip morphology when the stress increases: (a) crack tip morphology obtained under the moderate cyclic loading; (b) Morphology of the crack tip obtained with the diabolo sample under moderate cyclic loading (SEM photomicrograph); (c) the morphology of the crack tip evolves with the increase in stress. This image is the reference for the image correlation to obtain the kinematic field in the outlined zone; (d) A higher level of stress. This is the last image for the image correlation.

Figure 6. Image correlation in the outlined zone in Figure 5(b): (a) Cartography of the relative deformation in terms of the Green-Lagrange strain in direction 2; (b) variation of the relative deformation at points $\mathrm{A}, \mathrm{B}$, and $\mathrm{C}$ during stretching.

Figure 7. Mechanism of fatigue crack growth: front view: (a) crack tip morphology under moderate loading; (b) transition between the mechanism of fatigue crack growth under moderate loading and that 
under severe loading ( $\mathrm{c}$ and d) under severe fatigue loading, each striation is formed in one cycle.

Figure 8. Mechanism of fatigue crack growth: side view. 


\section{for Table of Contents use only}

The mechanism of fatigue crack growth in rubbers under severe loading: the effect of stress-induced crystallization

\section{Jean-Benoît Le Cam, Evelyne Toussaint}
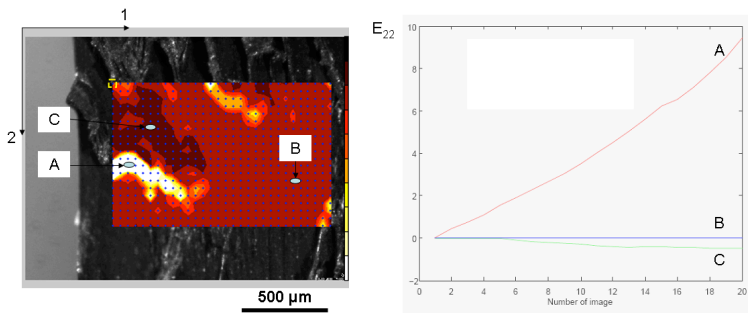

The relative deformation of zones located at the crack tip measured during stretching by means of digital image correlation. No deformation is observed in the more crystallized zone (see point B). 